\title{
DOA Estimation from Temporally and Spatially Correlated Narrowband Signals with Noncircular Sources
}

\author{
Sonia Ben Hassen, Faouzi Bellili, Abdelaziz Samet, and Sofiène Affes \\ INRS-EMT, 800, de la Gauchetière Ouest, Bureau 6900, Montreal, Qc, H5A 1K6, Canada \\ Emails:hassen@emt.inrs.ca, bellili@emt.inrs.ca, abdelaziz.samet@ept.rnu.tn, affes@emt.inrs.ca
}

\begin{abstract}
In this paper, we develop for the first time a method of estimating the DOA parameters assuming noncircular and spatially and temporally correlated signals. The new approach is based on the two-sided IV-SSF method (instrumental variable signal with subspace fitting). It will be shown that our newly developed method outperforms the classical two-sided IV-SSF in terms of lower bias and error variance. Its performance improvement increases as the noncircularity rate increases. Moreover, this improvement is more prominent at low SNR values. We also derive for the first time an analytical expression for the stochastic Cramér-Rao bound (CRB) of the DOA estimates from spatially and temporally correlated signals generated from noncircular sources. The new CRB is compared to that of circular and temporally correlated signals. It will be shown that the CRB obtained assuming both noncircular sources and temporally correlated signals is lower than the CRB derived considering only the temporal correlation. This illustrates the potential gain that both the noncircularity and the temporal correlation provide when considered together.
\end{abstract}

\section{INTRODUCTION}

The problem of estimating the direction-of-arrival (DOA) for multiple plane waves impinging on an arbitrary array of sensors has received considerable attention in array signal processing over the last few decades. As a consequence of this research effort, many DOA estimators have been extensively studied, assuming different data models. Indeed, in a standard DOA estimation model, snapshots are assumed to be independent and identically distributed (iid). For this model, several methods have been intensively studied assuming the signals to be generated from circular sources. The well-known estimators derived in this case are the deterministic (or conditional) and the unconditional maximum likelihood (ML) estimators [1], the MUltiple SIgnal Classification (MUSIC) estimator [2, 3] and the Method Of DOA Estimation (MODE) technique [4].

Unfortunately, the iid assumption considered in the aforementioned estimators pauses a challenging limitation on the applicability of the results in the real world and results in some practical difficulties. Therefore, efforts have been directed to considering more realistic models assuming the signals to be temporally correlated and generated from circular sources. In this context, two methods based on an instrumental variable (IV) approach were developed assuming the signals to be temporally correlated and circular Gaussian distributed, in the situation of unknown noise spatial covariance matrix $[5,6]$.
Yet, noncircular complex signals, for example binary phase shift keying (BPSK) and offset-quadrature-phase-shift-keying (OQPSK) modulated signals, are frequently encountered in digital communications. Therefore, more recently, some works have dealt with the derivation of new algorithms that exploit the noncircularity property of the signals $[7,8]$. These works show the potential benefit related to the noncircularity property. More recently, Haddadi, Nayebi and Aref have proposed in [9] a new algorithm which presents an improved version of the method developed in [6], but still for circular signals. But to the best of our knowledge, no contributions have dealt so far with the problem of estimating the DOA assuming the signals to be both spatially and temporally correlated and also generated from noncircular sources. Therefore, the aim of this work is to show the potential benefit of both temporal correlation and noncircularity of the signals considered together that could be gained in DOA estimation.

In fact, we propose for the first time an algorithm that extends the recent method developed in [9] assuming circular signals to the case of noncircular signals. Furthermore, we derive for the first time an explicit expression for the CRB of the DOA estimates from spatially and temporally correlated Gaussian distributed signals generated from noncircular sources.

This paper is organized as follows. In section II, we introduce the system model and assumptions. In section III, we formulate the newly proposed algorithm and discuss its statistical properties. In section IV, an explicit expression of the DOA CRB assuming spatially and temporally correlated signals generated from noncircular sources will be derived. In section $\mathrm{V}$, some simulation results will be presented and some concluding remarks will be drawn out.

Throughout this paper, matrices and vectors are represented by bold upper case and bold lower case characters, respectively. Vectors are, by default, in column orientation, while $(.)^{*},(.)^{T}$ and $(.)^{H}$ refer to conjugate, transpose and conjugate transpose, respectively. Moreover, $\mathrm{E}\{\},. \operatorname{tr}(),.\|.\|_{\text {Fro }}, \Re\{$.$\} and$ $\Im\{$.$\} stand for the expectation, trace, Frobenius norm, real$ and imaginary part operators, respectively. eig(.) represents the eigenvalues of a matrix. Finally, $\otimes$ and $\odot$ represent the Kronecker and the Hadamard-Schur product operators, respectively. 


\section{System Model}

Let an array of $L$ sensors receive the signals emitted by $K$ narrowband sources with unknown directions $\boldsymbol{\theta}=$ $\left[\theta_{1}, \ldots, \theta_{K}\right]$. Then, the received data can be modeled as a complex signal as follows:

$$
\boldsymbol{y}(t)=\boldsymbol{A} \boldsymbol{x}(t)+\boldsymbol{w}(t), \quad t=1,2, \ldots, N,
$$

where, $N$ represents the total number of received samples in the observation window. At time index $t, \boldsymbol{x}(t)$ is the sources' transmitted signals vector, $\boldsymbol{A}=\left[\boldsymbol{a}\left(\theta_{1}\right), \ldots, \boldsymbol{a}\left(\theta_{K}\right)\right]$ is the steering matrix with $\left\{\boldsymbol{a}\left(\theta_{i}\right)\right\}_{i=1, \ldots, K}$ are the array steering vectors parameterized by the scalar DOA parameters $\left\{\theta_{i}\right\}_{i=1, \ldots, K}$ and $\boldsymbol{w}(t)$ is the unknown noise vector. Stacking the received data over the whole observation window in a matrix $\boldsymbol{Y}$, (1) can be written in a matrix form as follows:

$$
\boldsymbol{Y}=\boldsymbol{A} \boldsymbol{X}+\boldsymbol{W},
$$

where $\boldsymbol{Y}=[\boldsymbol{y}(1), \ldots, \boldsymbol{y}(N)], \boldsymbol{X}=[\boldsymbol{x}(1), \ldots, \boldsymbol{x}(N)]$ and $\boldsymbol{W}=[\boldsymbol{w}(1), \ldots, \boldsymbol{w}(N)]$.

Now, assume that the transmitted signals $\boldsymbol{x}(t)$ are generated from noncircular sources. This means that $\mathrm{E}\left\{\boldsymbol{x}(t) \boldsymbol{x}^{T}(t)\right\} \neq \mathbf{0}$ contrarily to circular signals (where only $\mathrm{E}\left\{\boldsymbol{x} \boldsymbol{x}^{H}\right\} \neq 0$ ). Otherwise, the transmitted signals $\{\boldsymbol{x}(t)\}_{t=1,2, \ldots, N}$ are supposed to be zero-mean complex noncircular, temporally and possibly spatially correlated with conjugated and unconjugated covariance matrices $\mathcal{P}_{N K \times N K}$ and $\mathcal{P}_{N K \times N K}^{\prime}$, respectively defined as follows

$$
\begin{aligned}
& \mathcal{P}_{N K \times N K}=\operatorname{Block}_{i k}\left[\mathcal{P}_{K \times K}^{i k}\right], \\
& \mathcal{P}_{N K \times N K}^{\prime}=\operatorname{Block}_{i k}\left[\mathcal{P}_{K \times K}^{\prime i k}\right],
\end{aligned}
$$

with Block $_{i k}\left[\mathcal{P}_{K \times K}^{i k}\right]$ and Block $_{i k}\left[\mathcal{P}_{K \times K}^{i k}\right]$ representing block matrices with blocks $\mathcal{P}_{K \times K}^{i k}$ and $\mathcal{P}_{K \times K}^{\prime i k}$, respectively. The $i k^{\prime}$ th block of the block matrix $\mathcal{P}, \mathcal{P}^{i k}$, represents the first space-time covariance matrix of the signals and is defined as

$$
\mathcal{P}^{i k}=\mathrm{E}\left\{\boldsymbol{x}(i) \boldsymbol{x}^{H}(k)\right\} .
$$

Moreover, the $i k^{\prime}$ th block of the block matrix $\mathcal{P}^{\prime}, \mathcal{P}^{\prime i k}$, represents the second unconjugated spatial covariance matrix of the transmitted signals and is defined as

$$
\mathcal{P}^{\prime i k}=\mathrm{E}\left\{\boldsymbol{x}(i) \boldsymbol{x}^{T}(k)\right\} .
$$

The noise is assumed to be zero-mean Gaussian complex circular, possibly spatially correlated and temporally white with covariance matrix

$$
\mathcal{C}=\boldsymbol{I}_{N} \otimes \boldsymbol{C}(t) .
$$

where $\boldsymbol{C}(t)$ is defined by $\boldsymbol{C}(t)=\mathrm{E}\left\{\boldsymbol{w}(t) \boldsymbol{w}^{H}(t)\right\}$. Moreover, $\boldsymbol{I}_{p}$ is the $(p \times p)$ identity matrix. Consequently, the received signals are zero-mean complex noncircular, temporally and possibly spatially correlated with conjugated and unconjugated covariance matrices $\boldsymbol{\mathcal { R }}_{N L \times N L}$ and $\mathcal{R}_{N L \times N L}^{\prime}$, respectively, as follows

$$
\begin{aligned}
\mathcal{R}_{N L \times N L} & =\mathcal{A P} \mathcal{A}^{H}+\mathcal{C} \\
\mathcal{R}_{N L \times N L}^{\prime} & =\mathcal{A P} \mathcal{A}^{T},
\end{aligned}
$$

with

$$
\mathcal{A}=\boldsymbol{I}_{N} \otimes \boldsymbol{A} .
$$

In this paper, we consider the same assumptions $A 1, A 2$ and $A 3$ recently introduced in [6] as follows:

- A1) It is assumed that $K<L$ and that for any set of distinct DOA parameters $\theta_{1}, \ldots, \theta_{K}$, the vectors $\left\{\boldsymbol{a}\left(\theta_{1}\right), \ldots, \boldsymbol{a}\left(\theta_{K}\right)\right\}$ are linearly independent. Furthermore, $\boldsymbol{a}(\theta)$ is assumed to be differentiable with respect to $\theta$ and the true parameter vector $\boldsymbol{\theta}_{0}$ is an inner point of the set of parameter vectors of interest.

- A2) The transmitted signals $\{\boldsymbol{x}(t)\}_{t=1,2, \ldots, N}$ are assumed to be independent from the noise components $\{\boldsymbol{w}(t)\}_{t=1,2, \ldots, N}$.

- A3) Define the first cross-covariance matrix of the transmitted signals at time lag $k$ as follows:

$$
\boldsymbol{P}_{k}=\mathrm{E}\left\{\boldsymbol{x}(t-k) \boldsymbol{x}^{H}(t)\right\},
$$

and introduce the matrix of stacked first cross-covariances as follows

$$
\mathcal{J}=\left[\boldsymbol{P}_{-\frac{M}{2}}, \ldots, \boldsymbol{P}_{-1}, \boldsymbol{P}_{1}, \ldots, \boldsymbol{P}_{\frac{M}{2}}\right]^{T} .
$$

The signals are assumed to exhibit a "sufficient" temporal correlation so that no column of $\mathcal{J}$ is identically zero and so that the rank of $\mathcal{J}$, denoted $\mu$, satisfies $\mu>2 K-L$.

\section{Derivation Of The New DOA Estimation METHOD}

In this section, we propose a significant extension of the IVSSF method derived in [9] for circular signals to noncircular signals.

\section{A. Extended IV-SSF method}

Consider the two-sided instrumental variables vector defined in [9] by

$\phi(t)=\left[\boldsymbol{y}\left(t+\frac{M}{2}\right), \ldots, \boldsymbol{y}(t+1), \boldsymbol{y}(t-1), \ldots, \boldsymbol{y}\left(t-\frac{M}{2}\right)\right]^{T}$,

where $M$ is an even integer, superior to 2 . In order to take advantage of the signal noncircularity, we define the extended two-sided instrumental variables vector as follows:

$$
\widetilde{\phi}(t)=\left[\phi(t), \phi^{*}(t)\right]^{T} .
$$

Now, we introduce the first cross-covariance matrix of the transmitted signals at time lag $k$ as defined in (11) and the second cross-covariance matrix of the transmitted signals at time lag $k$ as follows:

$$
\boldsymbol{P}_{k}^{\prime}=\mathrm{E}\left\{\boldsymbol{x}(t-k) \boldsymbol{x}^{T}(t)\right\} .
$$

We also define the extended cross-covariance of the received data and the corresponding instrumental variable as follows:

$$
\widetilde{\boldsymbol{\Sigma}}=\mathrm{E}\left\{\widetilde{\boldsymbol{\phi}}(t) \widetilde{\boldsymbol{y}}^{H}(t)\right\},
$$

where $\widetilde{\boldsymbol{y}}(t)$ is the extended received vector defined as

$$
\widetilde{\boldsymbol{y}}(t)=\left[\boldsymbol{y}(t), \boldsymbol{y}^{*}(t)\right]^{T} \text {. }
$$


The extended cross-covariance matrix can then be written as

$$
\widetilde{\Sigma}=\left(\begin{array}{cc}
\Sigma & \Sigma^{\prime} \\
\Sigma^{\prime *} & \Sigma^{*}
\end{array}\right),
$$

where $\boldsymbol{\Sigma}=\mathrm{E}\left\{\boldsymbol{\phi}(t) \boldsymbol{y}^{H}(t)\right\}$ and $\boldsymbol{\Sigma}^{\prime}=\mathrm{E}\left\{\boldsymbol{\phi}(t) \boldsymbol{y}^{T}(t)\right\}$. Otherwise, $\boldsymbol{\Sigma}$ and $\boldsymbol{\Sigma}^{\prime}$ can be shown to verify

$$
\begin{aligned}
\boldsymbol{\Sigma} & =\mathcal{A}_{M} \mathcal{J} \boldsymbol{A}^{H}, \\
\boldsymbol{\Sigma}^{\prime} & =\mathcal{A}_{M} \mathcal{J}^{\prime} \boldsymbol{A}^{T},
\end{aligned}
$$

where $\mathcal{J}$ is defined in (12) and $\mathcal{A}_{M}$ and $\mathcal{J}^{\prime}$ are defined as follows

$$
\begin{aligned}
\mathcal{A}_{M} & =\boldsymbol{I}_{M} \otimes \boldsymbol{A}, \\
\mathcal{J}^{\prime} & =\left[\boldsymbol{P}_{-\frac{M}{2}}^{\prime}, \ldots, \boldsymbol{P}_{-1}^{\prime}, \boldsymbol{P}_{1}^{\prime}, \ldots, \boldsymbol{P}_{\frac{M}{2}}^{\prime}\right]^{T},
\end{aligned}
$$

with $\left\{\boldsymbol{P}_{k}^{\prime}\right\}_{k=-\frac{M}{2}}^{\frac{M}{2}}$ being defined in (14). Consequently, the extended cross covariance matrix of the received data and the corresponding instrumental variable can be simply written as:

$$
\widetilde{\boldsymbol{\Sigma}}=\widetilde{\mathcal{A}}_{M} \widetilde{\mathcal{J}} \widetilde{\boldsymbol{A}}^{H},
$$

where

$$
\begin{aligned}
\widetilde{\mathcal{A}}_{M} & =\left(\begin{array}{cc}
\mathcal{A}_{M} & \boldsymbol{0}_{L M \times K M} \\
\mathbf{0}_{L M \times K M} & \boldsymbol{A}_{M}{ }^{*}
\end{array}\right), \\
\widetilde{\mathcal{J}} & =\left(\begin{array}{cc}
\mathcal{J} & \mathcal{J}^{\prime} \\
\mathcal{J}^{\prime *} & \mathcal{J}^{*}
\end{array}\right), \\
\widetilde{\boldsymbol{A}} & =\left(\begin{array}{cc}
\boldsymbol{A}^{H} & \boldsymbol{0}_{K \times L} \\
\mathbf{0}_{K \times L} & \boldsymbol{A}^{T}
\end{array}\right),
\end{aligned}
$$

where $\mathbf{0}_{p \times q}$ represents the $(p \times q)$ null matrix. Moreover, we define the extended instrumental variable covariance matrix as follows

$$
\widetilde{\boldsymbol{\Phi}}=\mathrm{E}\left\{\widetilde{\boldsymbol{\phi}}(t) \widetilde{\boldsymbol{\phi}}^{H}(t)\right\}=\left(\begin{array}{cc}
\boldsymbol{\Phi} & \boldsymbol{\Phi}^{\prime} \\
\boldsymbol{\Phi}^{\prime *} & \boldsymbol{\Phi}^{*}
\end{array}\right),
$$

where $\boldsymbol{\Phi}=\mathrm{E}\left\{\boldsymbol{\phi}(t) \boldsymbol{\phi}^{H}(t)\right\}$ and $\boldsymbol{\Phi}^{\prime}=\mathrm{E}\left\{\boldsymbol{\phi}(t) \boldsymbol{\phi}^{T}(t)\right\}$. Consider here the assumption $A 3$. We denote $\mu^{\prime}$ the rank of $\widetilde{\mathcal{J}}$. Then, we have $\mu \leq \mu^{\prime} \leq 2 \mu$. Moreover, we consider the assumption $A 1$. The matrix $\boldsymbol{A}$ has a full rank. Therefore, the matrices $\mathcal{A}_{M}$ and $\widetilde{\mathcal{A}}_{M}$ have full ranks. Consequently, we have $\operatorname{rank}(\widetilde{\boldsymbol{\Sigma}})=\operatorname{rank}(\widetilde{\mathcal{J}})=\mu^{\prime}$. The singular value decomposition (SVD) of the matrix $\widetilde{\boldsymbol{\Phi}}^{-\frac{1}{2}} \widetilde{\boldsymbol{\Sigma}}$ yields the following result

$$
\widetilde{\boldsymbol{\Phi}}^{-\frac{1}{2}} \widetilde{\boldsymbol{\Sigma}}=\widetilde{\boldsymbol{U}}_{s} \widetilde{\boldsymbol{\Delta}}_{s} \widetilde{\boldsymbol{V}}_{s}^{H},
$$

where $\operatorname{rank}\left(\widetilde{\boldsymbol{\Delta}}_{s}\right)=\operatorname{rank}(\widetilde{\boldsymbol{\Sigma}})=\mu^{\prime}$. From (22) and (24), we show that the range space of $\widetilde{\boldsymbol{V}}_{s}$ is contained in the one of $\widetilde{\boldsymbol{A}}$. Consequently, there exists a full rank $2 K \times \mu^{\prime}$ matrix $\boldsymbol{T}$ such that

$$
\widetilde{\boldsymbol{V}}_{s}=\widetilde{\boldsymbol{A}}(\boldsymbol{\theta}) \boldsymbol{T} .
$$

Now, we define the sample estimates $\widehat{\widetilde{\Sigma}}$ and $\widehat{\widetilde{\Phi}}$ as follows:

$$
\begin{aligned}
& \widehat{\widetilde{\boldsymbol{\Sigma}}}=\frac{1}{N-M} \sum_{t=\frac{M}{2}+1}^{N-\frac{M}{2}} \widetilde{\phi}(t) \widetilde{\boldsymbol{y}}^{H}(t), \\
& \widehat{\widetilde{\boldsymbol{\Phi}}}=\frac{1}{N-M} \sum_{t=\frac{M}{2}+1}^{N-\frac{M}{2}} \widetilde{\phi}(t) \widetilde{\boldsymbol{\phi}}^{H}(t) .
\end{aligned}
$$

The SVD of the matrix $\widehat{\widetilde{\mathbf{\Phi}}}^{-\frac{1}{2}} \widehat{\widetilde{\mathbf{\Sigma}}}$ yields the following result

$$
\widehat{\widetilde{\boldsymbol{\Phi}}}^{-\frac{1}{2}} \widehat{\widetilde{\boldsymbol{\Sigma}}}=\widehat{\widetilde{\boldsymbol{U}}}_{s} \widehat{\widetilde{\boldsymbol{\Delta}}}_{s} \widehat{\widetilde{\boldsymbol{V}}}_{s}^{H}+\widehat{\widetilde{\boldsymbol{U}}}_{n} \widehat{\widetilde{\boldsymbol{\Delta}}}_{n} \widehat{\widetilde{\boldsymbol{V}}}_{n}^{H},
$$

where the matrix $\widehat{\widetilde{\Delta}}_{s}$ contains the $\mu^{\prime}$ largest singular values. Therefore, we obtain, similarly to the result obtained in [6], the following separable least-squares problem

$$
\{\widehat{\boldsymbol{\theta}}, \widehat{\boldsymbol{T}}\}=\arg \min _{\boldsymbol{\theta}, \boldsymbol{T}}\left\|\widehat{\widetilde{\boldsymbol{C}}}^{-\frac{1}{2}}\left(\widehat{\widetilde{\boldsymbol{V}}}_{s} \widehat{\widetilde{\boldsymbol{\Delta}}}_{s}-\widetilde{\boldsymbol{A}} \boldsymbol{T}\right)\right\|_{\text {Fro }}^{2},
$$

where

$$
\widetilde{\boldsymbol{C}}=\left(\begin{array}{cc}
\boldsymbol{C} & \mathbf{0}_{L \times L} \\
\mathbf{0}_{L \times L} & \boldsymbol{C}^{*}
\end{array}\right) .
$$

Minimizing (29) with respect to $\boldsymbol{T}$ yields the following criterion function:

$$
\widehat{\boldsymbol{\theta}}=\arg \min _{\boldsymbol{\theta}} V(\boldsymbol{\theta})
$$

where

$$
\begin{aligned}
& V(\boldsymbol{\theta})=\operatorname{tr}\left(\widehat{\widetilde{\boldsymbol{\Pi}}}_{0}^{\perp} \widehat{\widetilde{\boldsymbol{C}}}^{-\frac{1}{2}} \widehat{\widetilde{\boldsymbol{V}}}_{s} \widehat{\widetilde{\boldsymbol{\Delta}}}_{s}^{2} \widehat{\widetilde{\boldsymbol{V}}}_{s}^{H} \widehat{\widetilde{\boldsymbol{C}}}^{-\frac{1}{2}}\right), \\
& \widehat{\widetilde{\boldsymbol{\Pi}}}_{0}^{\perp}=\boldsymbol{I}_{2 L}-\widehat{\widetilde{\boldsymbol{C}}}^{-\frac{1}{2}} \widetilde{\boldsymbol{A}}\left(\widetilde{\boldsymbol{A}}^{H} \widehat{\widetilde{\boldsymbol{C}}}^{-1} \widetilde{\boldsymbol{A}}\right)^{-1} \widetilde{\boldsymbol{A}}^{H} \widehat{\widetilde{\boldsymbol{C}}}^{-\frac{1}{2}}
\end{aligned}
$$

This criterion is shown to be statistically equivalent to:

$$
\widehat{\boldsymbol{\theta}}=\arg \min _{\boldsymbol{\theta}} \operatorname{tr}\left(\widehat{\widetilde{\boldsymbol{\Pi}}}^{\perp} \widehat{\widetilde{\boldsymbol{R}}}_{0}^{-\frac{1}{2}} \widehat{\widetilde{\boldsymbol{V}}}_{s} \widehat{\widetilde{\boldsymbol{\Delta}}}_{s}^{2} \widehat{\widetilde{\boldsymbol{V}}}_{s}^{H} \widehat{\widetilde{\boldsymbol{R}}}_{0}^{-\frac{1}{2}}\right),
$$

where

$$
\widehat{\widetilde{\boldsymbol{\Pi}}}^{\perp}=\boldsymbol{I}_{2 L}-\widehat{\widetilde{\boldsymbol{R}}}_{0}^{-\frac{1}{2}} \widetilde{\boldsymbol{A}}\left(\widetilde{\boldsymbol{A}}^{H} \widehat{\widetilde{\boldsymbol{R}}}_{0}^{-1} \widetilde{\boldsymbol{A}}\right)^{-1} \widetilde{\boldsymbol{A}}^{H}{\widetilde{\widetilde{\boldsymbol{R}}}_{0}^{-\frac{1}{2}}}^{-}
$$

$\widehat{\widetilde{\boldsymbol{R}}}_{0}$ represents an estimate of $\widetilde{\boldsymbol{R}}_{0}=\mathrm{E}\left\{\widetilde{\boldsymbol{y}}(t) \widetilde{\boldsymbol{y}}^{H}(t)\right\}$.

\section{B. Statistical properties}

The minimizer of (31) converges with probability one (w.p.1) to the true parameter vector $\boldsymbol{\theta}_{0}$. Moreover, from standard statistical theory, the asymptotic error covariance matrix is given by this following expression

$$
\boldsymbol{C}_{v}=\boldsymbol{H}^{-1} \boldsymbol{Q} \boldsymbol{H}^{-1},
$$

where

$$
\begin{aligned}
\boldsymbol{H} & =\lim _{N \rightarrow+\infty} V^{\prime \prime}\left(\boldsymbol{\theta}_{0}\right), \quad \text { (w.p.1) } \\
\boldsymbol{Q} & =\lim _{N \rightarrow+\infty} N \mathrm{E}\left\{V^{\prime}\left(\boldsymbol{\theta}_{0}\right) V^{\prime T}\left(\boldsymbol{\theta}_{0}\right)\right\},
\end{aligned}
$$

with $V^{\prime}($.$) and V^{\prime \prime}($.$) being the gradient of V($.$) previously$ defined in (32) with respect to $\boldsymbol{\theta}$ and the Hessian matrix, respectively. After some algebraic manipulations (see appendices A and B), we obtain the following expressions for $\boldsymbol{H}$ and $\boldsymbol{Q}$

$$
\begin{aligned}
& \boldsymbol{H}=4 \Re\left\{\left(\boldsymbol{D}^{H} \boldsymbol{C}^{-\frac{1}{2}} \boldsymbol{\Pi}_{0}{ }^{\perp} \boldsymbol{C}^{-\frac{1}{2}} \boldsymbol{D}\right)\right.
\end{aligned}
$$

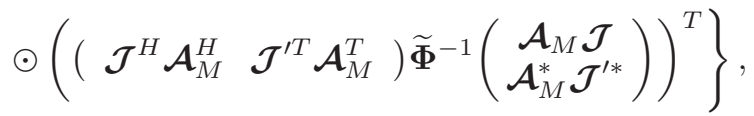

$$
\begin{aligned}
& \boldsymbol{Q}=2 \boldsymbol{H} .
\end{aligned}
$$


Consequently, the asymptotic covariance matrix of $\boldsymbol{\theta}$ is given by

$$
\begin{aligned}
& \boldsymbol{C}_{v}=\frac{1}{2}\left[\Re \left\{\left(\boldsymbol{D}^{H} \boldsymbol{C}^{-\frac{1}{2}} \boldsymbol{\Pi}_{0}^{\perp} \boldsymbol{C}^{-\frac{1}{2}} \boldsymbol{D}\right)\right.\right. \\
& \odot\left(\begin{array}{ll}
\left(\mathcal{J}^{H} \mathcal{A}_{M}^{H}\right. & \left.\left.\left.\left.\mathcal{J}^{\prime T} \mathcal{A}_{M}^{T}\right) \widetilde{\boldsymbol{\Phi}}^{-1}\left(\begin{array}{c}
\mathcal{A}_{M} \mathcal{J} \\
\mathcal{A}_{M}^{*} \mathcal{J}^{\prime *}
\end{array}\right)\right)^{T}\right\}\right]^{-1}
\end{array}\right.
\end{aligned}
$$

For circular signals, we have $\mathcal{J}^{\prime}=\mathbf{0}_{K M \times K}$ and $\boldsymbol{\Phi}^{\prime}=$ $\mathbf{0}_{L M \times L M}$. Therefore, (41) reduces to

$$
\begin{aligned}
\boldsymbol{C}_{v}^{\mathrm{cir}}= & \frac{1}{2}\left[\Re \left\{\left(\boldsymbol{D}^{H} \boldsymbol{C}^{-\frac{1}{2}} \boldsymbol{\Pi}_{0}^{\perp} \boldsymbol{C}^{-\frac{1}{2}} \boldsymbol{D}\right) \odot\right.\right. \\
& \left.\left.\left(\mathcal{J}^{H} \mathcal{A}_{M}^{H} \boldsymbol{\Phi}^{-1} \mathcal{A}_{M} \mathcal{J}\right)^{T}\right\}\right]^{-1},
\end{aligned}
$$

derived in [9] as a special case of our general method. Applying [1, lemma A.4], we prove, thanks to standard results of linear algebra (see [10, App. A, result R.19]), the following inequality

$$
C_{v}^{\text {noncir }} \leq C_{v}^{\text {cir }},
$$

where $\boldsymbol{A}-\boldsymbol{B} \geq \mathbf{0}$ means that $\boldsymbol{A}-\boldsymbol{B}$ is definite positive.

IV. NEW CRB FOR NONCIRCULAR GAUSSIAN DISTRIBUTED AND TEMPORALLY CORRELATED SIGNALS

To derive the CRB of the considered model, we assume that the noise is circular Gaussian distributed and the noise covariance matrix $C$ is known (possibly up to a multiplicative scalar). Then, we define the parameter vector as follows

$$
\boldsymbol{\alpha}=\left(\boldsymbol{\theta}^{T}, \boldsymbol{\beta}^{T}\right)^{T},
$$

where $\theta$, introduced in section II, represents the directions of the narrowband sources and $\boldsymbol{\beta}$ is defined by

$$
\begin{aligned}
\boldsymbol{\beta}= & \left(\left(\Re\left\{\boldsymbol{P}_{i j}\right\}, \Im\left\{\boldsymbol{P}_{i j}\right\}, \Re\left\{\boldsymbol{P}_{i j}^{\prime}\right\}, \Im\left\{\boldsymbol{P}_{i j}^{\prime}\right\}\right)_{1 \leq j<i \leq N K},\right. \\
& \left.\left(\boldsymbol{P}_{i i}, \Re\left\{\boldsymbol{P}_{i i}^{\prime}\right\}, \Im\left\{\boldsymbol{P}_{i i}^{\prime}\right\}\right)_{i=1, \ldots, N K}\right)^{T} .
\end{aligned}
$$

We also define the vector $\overline{\boldsymbol{y}}_{N}$ as

$$
\overline{\boldsymbol{y}}_{N}=\left[\boldsymbol{C}^{-\frac{1}{2}} \boldsymbol{y}(1), \ldots, \boldsymbol{C}^{-\frac{1}{2}} \boldsymbol{y}(N)\right]^{T} .
$$

Moreover, we introduce the following extended vector $\widetilde{\bar{y}}_{N}$

$$
\widetilde{\overline{\boldsymbol{y}}}_{N}=\left[\overline{\boldsymbol{y}}_{N}, \overline{\boldsymbol{y}}_{N}^{*}\right]^{T} \text {. }
$$

Then, we have $\widetilde{\overline{\boldsymbol{y}}}_{N} \sim \mathcal{N}\left(\mathbf{0}, \widetilde{\boldsymbol{\mathcal { R }}}_{N}\right)$ where $\widetilde{\boldsymbol{\mathcal { R }}}_{N}=\mathrm{E}\left\{\widetilde{\boldsymbol{y}}_{N} \widetilde{\overline{\boldsymbol{y}}}_{N}^{H}\right\}=$ $\widetilde{\bar{G}} \widetilde{\mathcal{P}} \widetilde{\bar{G}}^{H}+\boldsymbol{I}_{2 N L}$ with

$$
\begin{aligned}
\widetilde{\bar{G}} & =\left(\begin{array}{cc}
\overline{\boldsymbol{G}} & \boldsymbol{0}_{N L \times N K} \\
\boldsymbol{0}_{N L \times N K} & \overline{\boldsymbol{G}}^{*}
\end{array}\right), \\
\widetilde{\mathcal{P}} & =\left(\begin{array}{cc}
\mathcal{P} & \mathcal{P}^{\prime} \\
\mathcal{P}^{\prime *} & \mathcal{P}^{*}
\end{array}\right),
\end{aligned}
$$

with $\mathcal{P}$ and $\mathcal{P}^{\prime}$ being previously defined in (3) and (4), respectively, and $\overline{\boldsymbol{G}}=\boldsymbol{I}_{N} \otimes\left(\boldsymbol{C}^{-\frac{1}{2}} \boldsymbol{A}\right)$. Following the same steps of [11], we obtain the following expression of $[\mathrm{CRB}]_{i k}^{-1}$

$$
[\mathrm{CRB}]_{i k}^{-1}=\Re\left\{\operatorname{tr}\left(\widetilde{\bar{G}}_{i}^{H} \Pi_{\widetilde{\widetilde{G}}^{\perp}}^{\perp} \widetilde{\bar{G}}_{k} \widetilde{\mathcal{P}} \widetilde{\bar{G}}^{H} \widetilde{\mathcal{R}}_{N}^{-1} \widetilde{\bar{G}} \widetilde{\mathcal{P}}\right)\right\},
$$

where $\widetilde{\bar{G}}_{i}=\frac{\partial \widetilde{\widetilde{\boldsymbol{G}}}(\boldsymbol{\theta})}{\partial \theta_{i}}$ and $\boldsymbol{\Pi}_{\tilde{\bar{G}}^{\perp}}^{\perp}=\boldsymbol{I}_{2 N L}-\widetilde{\overline{\boldsymbol{G}}}\left(\widetilde{\overline{\boldsymbol{G}}}^{H} \widetilde{\bar{G}}^{-1} \widetilde{\overline{\boldsymbol{G}}}^{H}\right.$. After tedious algebraic manipulations, we obtain the following expression of $\operatorname{CRB}(\boldsymbol{\theta})$ for temporally correlated signals generated from noncircular sources

$$
\begin{aligned}
& \mathrm{CRB}^{\text {noncir/cor }}(\boldsymbol{\theta})=\frac{1}{2}\left[\Re \left\{\left(\boldsymbol{D}^{H} \boldsymbol{C}^{-\frac{1}{2}} \boldsymbol{\Pi}_{\boldsymbol{G}}^{\perp} \boldsymbol{C}^{-\frac{1}{2}} \boldsymbol{D}\right) \odot\right.\right.
\end{aligned}
$$

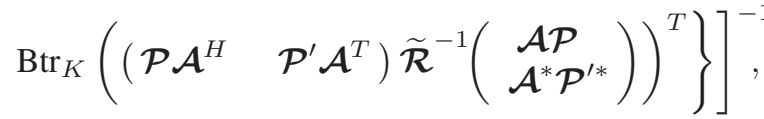

where $\mathcal{A}$ is defined in (10) and $\mathrm{Btr}_{K}$ denotes the block trace defined by $\operatorname{Btr}_{K}(\mathcal{P})=\sum_{i} \mathcal{P}_{K \times K}^{i i}$.

\section{Graphical Representations}

In this section, we present some graphical representations to show the performance of our new extended version of the classical two-sided IV-SSF method and to illustrate the new CRB bounds for comparisons.

Throughout this section, we consider a complex noncircular Gaussian signal with noncircularity rate $\rho$ and noncircularity phase $\phi=\pi / 2$. This signal impinges on a uniform linear array of 4 sensors separated by a half-wavelength. We consider a single source (for the sake of simplicity) located at $\theta=0.2$ radians with respect to the normal of the array broadside. The number of instrumental variables is set to $M=2$. Moreover, 10000 independent simulation runs have been performed to obtain the empirical performance measures. The temporal correlation of the signal is simulated via filtering the complex noncircular Gaussian signal with an FIR filter with relative tap weights

$$
f(z)=1+0.5 z^{-1}+0.3 z^{-2}+0.2 z^{-3}+0.1 z^{-4},
$$

which is then normalized to give a unit-energy filter. The estimates are calculated using a coarse search with grid size 0.001 .

In Fig. 1, the bias and the standard deviation of the estimates of $\theta$ of our extended two-sided IV-SSF method are presented versus the SNR for $N=100$ snapshots and compared to those of the two-sided IV-SSF method derived in [9] for circular sources (referred to as circular method). We see from this figure that the extended two-sided IV-SSF outperforms the circular method in terms of both lower bias and lower standard deviation. The improvement made by our extended two-sided IV-SSF is more pronounced at low SNR values.

We also see from Fig. 1 that the bias becomes negligible, especially at high SNR values for both versions. Therefore, it is still rewarding to compare the performance of the extended and the circular methods to the square root of the CRB ${ }^{\text {noncir/cor }}$ and $\mathrm{CRB}^{\mathrm{cir} / \mathrm{cor}}$, respectively, which is depicted also in Fig. 1. To represent these CRBs, we define the first and the second (unconjugated) signal space-time covariance matrices by $\mathcal{P}=\boldsymbol{P}_{t} \otimes \boldsymbol{P}$ and $\mathcal{P}^{\prime}=\boldsymbol{P}_{t} \otimes \boldsymbol{P}^{\prime}$, respectively, where $\boldsymbol{P}$ and $\boldsymbol{P}^{\prime}$ are the covariance matrices of the noncircular complex Gaussian signal. Moreover, $\boldsymbol{P}_{t}$ represents the temporal 


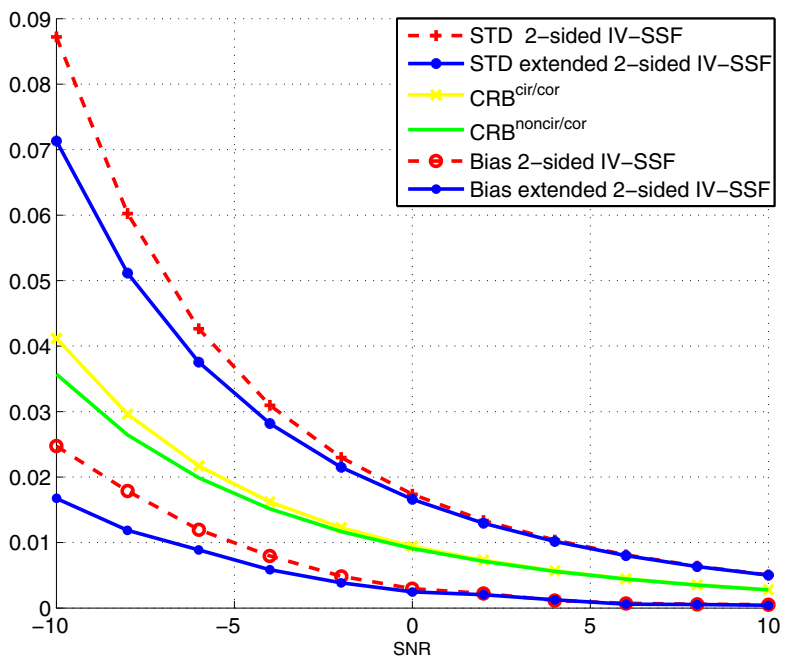

Figure 1. Bias and standard deviation of the extended and circular two-sided IV-SSF methods versus the SNR, for $N=100$ and $\rho=1$.

correlation matrix computed using (43). We verify that

$$
\begin{aligned}
\mathrm{CRB}^{\text {noncir/cor }} & <\mathrm{STD}^{\text {extended method }}, \\
\mathrm{CRB}^{\text {cir/cor }} & <\mathrm{STD}^{\text {circular method }} .
\end{aligned}
$$

We also show that $\mathrm{CRB}^{\text {noncir/cor }}<\mathrm{CRB}^{\text {cir/cor }}$. This illustrates the potential gain that both the noncircularity and the temporal correlation offer when considered together. Now, in Fig. 2, the bias and the standard deviations of the estimates of $\theta$ of the two methods are presented versus the number of snapshots $N$ for $\mathrm{SNR}=0 \mathrm{~dB}$ and $\rho=1$. It can be seen from Fig. 2 that the extended two-sided

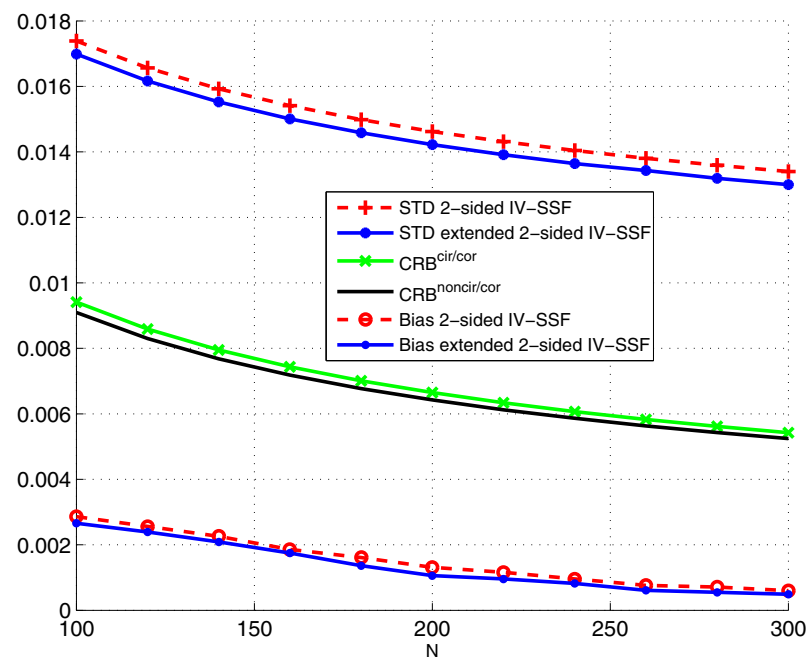

Figure 2. Bias and standard deviation of extended and circular two-sided IV-SSF methods versus the number of snapshots, for $\mathrm{SNR}=0 \mathrm{~dB}$ and $\rho=1$.

IV-SSF outperforms the classical circular method especially in terms of standard deviation. The improvement made by the extended method is constant with the number of snapshots $N$. Finally, Fig. 3 represents the ratio $r_{1}=$
STD ${ }^{\text {Extended two-sided IV-SSF method }} /$ STD $^{\text {Circular two-sided IV-SSF method }}$ as a function of the noncircularity rate $\rho$. We notice from this

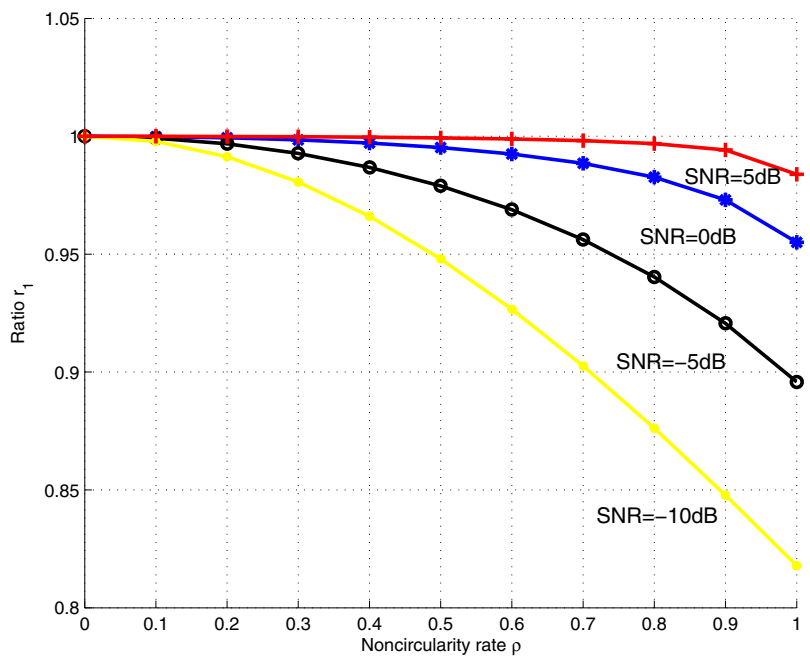

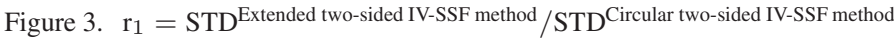
as a function of the noncircularity rate $\rho$, for $\mathrm{SNR}=0 \mathrm{~dB}$ and $N=100$.

figure that the improvement made by the extended two-sided IV-SSF method increases as the noncircularity rate increases. Moreover, this improvement is more prominent at low SNR values.

\section{CONCLUSION}

In this paper, we developed for the first time a method for estimating the DOA parameters assuming noncircular and spatially and temporally correlated signals. The new proposed method extends the well known two-sided IV-SSF approach to noncircular and time-correlated signals. We proved that our newly proposed method outperforms the classical two-sided IV-SSF method in terms of lower bias and error variance. We also derived for the first time an explicit expression for the stochastic Cramér-Rao bound (CRB) of the DOA estimates for spatially and temporally correlated signals generated from noncircular sources. This CRB was compared to that of circular temporally correlated signals. We showed the potential gain that both the noncircularity and the temporal correlation provide when considered together.

\section{APPENDIX A - DERIVATION OF $\boldsymbol{H}$}

Starting from (37), we obtain similarly to [6] the following expression for the $i k$, th entry of the limiting Hessian matrix

$$
\boldsymbol{H}_{i k}=2 \Re\left\{\operatorname{tr}\left(\widetilde{\boldsymbol{D}}_{i}^{H} \widetilde{\boldsymbol{C}}^{-\frac{1}{2}} \widetilde{\boldsymbol{\Pi}}_{0}^{\perp} \widetilde{\boldsymbol{C}}^{-\frac{1}{2}} \widetilde{\boldsymbol{D}}_{k} \widetilde{\boldsymbol{A}}^{\dagger} \widetilde{\boldsymbol{V}}_{s} \widetilde{\boldsymbol{\Delta}}_{s}^{2} \widetilde{\boldsymbol{V}}_{s}^{H} \widetilde{\boldsymbol{A}}^{\dagger H}\right)\right\}
$$

where

$$
\begin{aligned}
\widetilde{\boldsymbol{D}}_{i} & =\left(\begin{array}{cc}
\boldsymbol{D}_{i} & \mathbf{0}_{L \times K} \\
\mathbf{0}_{L \times K} & \boldsymbol{D}_{i}^{*}
\end{array}\right), \\
\boldsymbol{D}_{i} & =\partial \boldsymbol{A}(\boldsymbol{\theta}) / \partial \theta_{i}, \\
\widetilde{\boldsymbol{A}}^{\dagger} & =\left(\widetilde{\boldsymbol{A}}^{H} \widetilde{\boldsymbol{A}}\right)^{-1} \widetilde{\boldsymbol{A}}^{H} .
\end{aligned}
$$


We also exploit the particular structure of $\widetilde{C}$ and $\widetilde{\boldsymbol{\Pi}}_{0}^{\perp}$. Therefore, we obtain after tedious algebraic manipulations the following expression of $[\boldsymbol{H}]_{i k}$

$$
\begin{aligned}
{[\boldsymbol{H}]_{i k}=} & 2 \Re\left\{\operatorname { t r } \left(\left(\begin{array}{cc}
\boldsymbol{D}_{i}^{H} \boldsymbol{C}^{-\frac{1}{2}} \boldsymbol{\Pi}_{0}^{\perp} \boldsymbol{C}^{-\frac{1}{2}} \boldsymbol{D}_{k} & \mathbf{0}_{K \times K} \\
\mathbf{0}_{K \times K} & \left(\boldsymbol{D}_{i}^{H} \boldsymbol{C}^{-\frac{1}{2}} \boldsymbol{\Pi}_{0}^{\perp} \boldsymbol{C}^{-\frac{1}{2}} \boldsymbol{D}_{k}\right)^{*}
\end{array}\right) \times\right.\right. \\
& \left.\left.\widetilde{\mathcal{J}}^{H} \widetilde{\mathcal{A}}_{M}^{H} \widetilde{\boldsymbol{\Phi}}^{-1} \widetilde{\mathcal{A}}_{M} \widetilde{\mathcal{J}}\right)\right\} .
\end{aligned}
$$

Now, we consider $\mathcal{J}=\left(\boldsymbol{C}_{l 1}, \ldots, \boldsymbol{C}_{l i}, \ldots, \boldsymbol{C}_{l K}\right)$ and $\mathcal{J}^{\prime}=\left(\boldsymbol{C}_{l 1}^{\prime}, \ldots, \boldsymbol{C}_{l i}^{\prime}, \ldots, \boldsymbol{C}_{l K}^{\prime}\right)$ where $\left\{\boldsymbol{C}_{l i}\right\}_{K M \times 1}$ and $\left\{\boldsymbol{C}_{l i}^{\prime}\right\}_{K M \times 1}$ are the $i^{\text {th }}$ columns of $\mathcal{J}$ and $\mathcal{J}^{\prime}$, respectively. We also consider $\boldsymbol{D}=\left(\boldsymbol{d}_{1}, \ldots, \boldsymbol{d}_{K}\right)$ where $\boldsymbol{d}_{i}=$ $\partial \boldsymbol{a}\left(\theta_{i}\right) / \partial \theta_{i}, \quad i=1,2, \ldots, K$. Then, $\boldsymbol{D}_{i}$ can be written as $\boldsymbol{D}_{i}=\left(\mathbf{0}_{L \times 1}, \ldots, \boldsymbol{d}_{i}, \ldots, \mathbf{0}_{L \times 1}\right)$. Therefore, we obtain after tedious algebraic manipulations the following expression of $[\boldsymbol{H}]_{i j}$

$$
\begin{aligned}
& {[\boldsymbol{H}]_{i k}=4 \Re\left\{\left(\boldsymbol{d}_{i}^{H} \boldsymbol{C}^{-\frac{1}{2}} \boldsymbol{\Pi}_{0}^{\perp} \boldsymbol{C}^{-\frac{1}{2}} \boldsymbol{d}_{k}\right)\left(C_{l k}^{H} \quad \boldsymbol{C}_{l k}^{\prime T}\right) \times\right.} \\
& \left.\left(\begin{array}{lr}
\mathcal{A}_{M}^{H} & \mathbf{0}_{K M \times L M} \\
\mathbf{0}_{K M \times L M} & \boldsymbol{\mathcal { A }}_{M}^{T}
\end{array}\right) \widetilde{\boldsymbol{\Phi}}^{-1}\left(\begin{array}{ll}
\mathcal{A}_{M} & \mathbf{0}_{L M \times K M} \\
\mathbf{0}_{L M \times K M} & \mathcal{A}_{M}^{*}
\end{array}\right)\left(\begin{array}{c}
\boldsymbol{C}_{l i} \\
\boldsymbol{C}_{l i}^{\prime *}
\end{array}\right)\right\} .
\end{aligned}
$$

Consequently, we obtain the expression of $\boldsymbol{H}$ as given by (39).

\section{APPENDIX B - DERIVATION OF $\boldsymbol{Q}$}

We denote by $\boldsymbol{V}_{i}(\boldsymbol{\theta})$ the derivative of $\boldsymbol{V}(\boldsymbol{\theta})$ with respect to $\theta_{i}$. Similarly to [6], $\boldsymbol{V}_{i}(\boldsymbol{\theta})$ can be approximated by

$$
\boldsymbol{V}_{i}(\boldsymbol{\theta}) \simeq-2 \Re\left\{\operatorname{tr}\left(\widetilde{\boldsymbol{D}}_{i}^{H} \widetilde{\boldsymbol{C}}^{-\frac{1}{2}} \widetilde{\boldsymbol{\Pi}}_{0}^{\perp} \widetilde{\boldsymbol{C}}^{-\frac{1}{2}} \widetilde{\boldsymbol{X}}_{N} \widetilde{\boldsymbol{\Phi}}^{-1} \widetilde{\boldsymbol{\Sigma}} \widetilde{\boldsymbol{A}}^{\dagger H}\right)\right\},
$$

where

$$
\widetilde{\boldsymbol{X}}_{N}=\frac{1}{n-M} \sum_{t=\frac{M}{2}+1}^{n-\frac{M}{2}} \widetilde{\boldsymbol{w}}(t) \widetilde{\phi}^{H}(t)=\left(\begin{array}{cc}
\boldsymbol{X}_{N} & \boldsymbol{X}_{N}^{\prime} \\
\boldsymbol{X}_{N}^{\prime *} & \boldsymbol{X}_{N}^{*}
\end{array}\right),
$$

with

$$
\begin{aligned}
\boldsymbol{X}_{N} & =\frac{1}{n-M} \sum_{t=\frac{M}{2}+1}^{n-\frac{M}{2}} \boldsymbol{w}(t) \boldsymbol{\phi}^{H}(t), \\
\boldsymbol{X}_{N}^{\prime} & =\frac{1}{n-M} \sum_{t=\frac{M}{2}+1}^{n-\frac{M}{2}} \boldsymbol{w}(t) \boldsymbol{\phi}^{T}(t) .
\end{aligned}
$$

Some algebraic manipulations yield the following expression of $\boldsymbol{V}_{i}(\boldsymbol{\theta})$

$$
\boldsymbol{V}_{i}(\boldsymbol{\theta}) \simeq-4 \Re\left\{\left(\boldsymbol{\alpha}_{i}^{H} \boldsymbol{X}_{N} \boldsymbol{h}_{i}+\boldsymbol{\alpha}_{i}^{H} \boldsymbol{X}_{N}^{\prime} \boldsymbol{g}_{i}\right)\right\} .
$$

where $\boldsymbol{\alpha}_{i}$ is the $i^{\text {th }}$ column of the matrix $\boldsymbol{C}^{-\frac{1}{2}} \boldsymbol{\Pi}_{0}^{\perp} \boldsymbol{C}^{-\frac{1}{2}} \boldsymbol{D}$ and $\boldsymbol{h}_{i}$ and $\boldsymbol{g}_{i}$ are the $i^{\text {th }}$ columns of the block matrices $\left(\widetilde{\boldsymbol{\Phi}}^{-1} \widetilde{\boldsymbol{\Sigma}} \widetilde{\boldsymbol{A}}^{\dagger H}\right)_{(1: M L, 1: K)}$ and $\left(\widetilde{\boldsymbol{\Phi}}^{-1} \widetilde{\boldsymbol{\Sigma}} \widetilde{\boldsymbol{A}}^{\dagger H}\right)_{(1: M L, K+1: 2 K)}$, respectively. Using some properties of the real part operator, we prove after tedious algebraic manipulations, the following expression of $\mathrm{E}\left\{\boldsymbol{V}_{i}(\boldsymbol{\theta}) \boldsymbol{V}_{j}(\boldsymbol{\theta})\right\}$

$$
\begin{gathered}
\mathrm{E}\left\{\boldsymbol{V}_{i}(\boldsymbol{\theta}) \boldsymbol{V}_{j}(\boldsymbol{\theta})\right\}=\frac{8}{N} \Re\left\{\left(\boldsymbol{\alpha}_{i}^{H} \boldsymbol{C} \boldsymbol{\alpha}_{j}\right) \times\right. \\
\left.\left(\begin{array}{cc}
\boldsymbol{h}_{j}^{H} & \boldsymbol{g}_{j}^{H}
\end{array}\right) \widetilde{\boldsymbol{\Phi}}\left(\begin{array}{c}
\boldsymbol{h}_{i} \\
\boldsymbol{g}_{i}
\end{array}\right)\right\}+o\left(\frac{1}{N}\right) .
\end{gathered}
$$

Now, we have

$$
\begin{aligned}
\left(\boldsymbol{C}^{-\frac{1}{2}} \boldsymbol{\Pi}_{0}^{\perp} \boldsymbol{C}^{-\frac{1}{2}} \boldsymbol{D}\right)^{H} \boldsymbol{C}\left(\boldsymbol{C}^{-\frac{1}{2}} \boldsymbol{\Pi}_{0}^{\perp} \boldsymbol{C}^{-\frac{1}{2}} \boldsymbol{D}\right)= & \boldsymbol{D}^{H} \boldsymbol{C}^{-\frac{1}{2}} \boldsymbol{\Pi}_{0}^{\perp} \times \\
& \boldsymbol{C}^{-\frac{1}{2}} \boldsymbol{D} .
\end{aligned}
$$

We also have

$$
\begin{aligned}
& \left(\widetilde{\boldsymbol{\Phi}}^{-1} \widetilde{\boldsymbol{\Sigma}} \widetilde{\boldsymbol{A}}^{\dagger H}\right)^{H} \widetilde{\boldsymbol{\Phi}}\left(\widetilde{\boldsymbol{\Phi}}^{-1} \widetilde{\boldsymbol{\Sigma}} \widetilde{\boldsymbol{A}}^{\dagger H}\right)=\widetilde{\boldsymbol{A}}^{\dagger} \widetilde{\boldsymbol{\Sigma}}^{H} \widetilde{\boldsymbol{\Phi}}-1 \widetilde{\boldsymbol{\Sigma}} \widetilde{\boldsymbol{A}}^{\dagger H}, \\
= & \left(\begin{array}{cc}
\boldsymbol{A}^{\dagger} \boldsymbol{\Sigma}^{H} & \boldsymbol{A}^{\dagger} \boldsymbol{\Sigma}^{\prime T} \\
\boldsymbol{A}^{\dagger *} \boldsymbol{\Sigma}^{\prime H} & \boldsymbol{A}^{\dagger *} \boldsymbol{\Sigma}^{T}
\end{array}\right) \widetilde{\boldsymbol{\Phi}}^{-1}\left(\begin{array}{cc}
\boldsymbol{\Sigma} \boldsymbol{A}^{\dagger H} & \boldsymbol{\Sigma}^{\prime} \boldsymbol{A}^{\dagger T} \\
\boldsymbol{\Sigma}^{\prime *} \boldsymbol{A}^{\dagger H} & \boldsymbol{\Sigma}^{*} \boldsymbol{A}^{\dagger T}
\end{array}\right) .
\end{aligned}
$$

Consequently, from (38), (45), (45) and (46), we conclude that

$$
\begin{aligned}
& \boldsymbol{Q}=8 \Re\left\{\left(\boldsymbol{D}^{H} \boldsymbol{C}^{-\frac{1}{2}} \boldsymbol{\Pi}_{0}{ }^{\perp} \boldsymbol{C}^{-\frac{1}{2}} \boldsymbol{D}\right) \odot\right. \\
& \left.\left.\left(\begin{array}{ll}
\boldsymbol{A}^{\dagger} \boldsymbol{\Sigma}^{H} & \boldsymbol{A}^{\dagger} \boldsymbol{\Sigma}^{\prime T}
\end{array}\right] \widetilde{\boldsymbol{\Phi}}^{-1}\left(\begin{array}{c}
\boldsymbol{\Sigma} \boldsymbol{A}^{\dagger H} \\
\boldsymbol{\Sigma}^{* *} \boldsymbol{A}^{\dagger H}
\end{array}\right)\right)^{T}\right\} .
\end{aligned}
$$

Using (18) and (19) and some algebraic manipulations yield the expression of $Q$ as given by (40)

\section{REFERENCES}

[1] P. Stoica and A. Nehorai, "Performance Study of Conditional and Unconditional Direction-of-Arrival Estimation," IEEE Trans. Acoust., Speech, Sig. Process., vol. 38, pp. 1783-1795, Oct. 1990.

[2] P. Stoica and A. Nehorai, "MUSIC, Maximum Likelihood, and Cramer-Rao Bound," IEEE Trans. Acoust., Speech, Sig. Process., vol. 37, pp. 720-741, May 1989.

[3] P. Stoica and A. Nehorai, "MUSIC, Maximum Likelihood, and Cramér-Rao Bound: Further Results and Comparisons," IEEE Trans. Acoust., Speech, Sig. Process., vol. 38, pp. 2140-2150, Dec. 1990.

[4] P. Stoica and K.C Sharman, "Maximum Likelihood Methods for Direction-of-Arrival Estimation, "IEEE Trans. Acoust., Speech, Sig. Process., vol. 38, pp. 1132-1143, July 1990.

[5] P. Stoica, M. Viberg, and B. Ottersten "Instrumental Variable Approach to Array Processing in Spatially Correlated Noise Fields," IEEE Trans. Sig. Process., vol. 42, pp. 121-133, Jan. 1994.

[6] M. Viberg, P. Stoica, and B. Ottersten "Array Processing in Correlated Noise Fields Based on Instrumental Variables and Subspace Fitting," IEEE Trans. Sig. Process., vol. 43, pp. 11871199, May 1995.

[7] J. P. Delmas, "Asymptotically Minimum Variance Second-Order Estimation for Noncircular Signals with Application to DOA Estimation," IEEE Trans. Sig. Process., vol. 52, pp. 1235-1241, May 2004.

[8] H. Abeida and J. P. Delmas, "MUSIC-Like Estimation of Direction of Arrival for Noncircular Sources," IEEE Trans. Sig. Process., vol. 54, pp. 2678-2689, July 2006.

[9] F. Haddadi, M. M. Nayebi, and M. R. Aref, "Direction-ofArrival Estimation for Temporally Correlated Narrowband Signals," IEEE Trans. Sig. Process., vol. 57, pp. 600-609, Apr. 2009.

[10] P. Stoica and R. Moses, Introduction to Spectral Analysis. Upper Saddle River, NJ: Prentice-Hall, 1997.

[11] P. Stoica, E. G. Larsson, and A. B. Gershman, "The Stochastic CRB for Array Processing: A Textbook Derivation," IEEE Sig. Process. Lett., vol. 8, pp. 148-150, May 2001. 